\title{
An attempt to increase agility of garment industry
}

\begin{abstract}
Agility in the industry is an important issue in this competitive market. Agility ensures the rapid action plan execution in the firm and a suggested plan has been implemented in the industry. In this plan two excel file named "Line wise visual operator status" and "Skill matrix" have been included. In "Line wise visual operator status" file, the hourly updated status of sewing lines like absenteeism, low efficient operator, imperfect operator etc. are shown. And, through the "Skill matrix" it can be found out right operator for replacement and their performance. In the previous system of the industry it took about 75.61 minutes to execute a plan in the sewing line. But, after implementing the current attempt the total time for execution of action plan it takes 38.73 minutes. So, the industry can take the initiatives within 36.87 minutes faster than previous and it accelerates the agility of the industry.
\end{abstract}

Keywords: absenteeism, agile manufacturing system, industrial engineering (IE), line wise visual operator status, low efficient worker, skill matrix, sewing line.

\author{
Volume 5 Issue 3 - 2019
}

\author{
Shadman Ahmed Khan,' Tarikul Islam, ${ }^{2}$ Sazid \\ Elahi, ${ }^{3}$ Md Newaj Sharif,' Md Miraj Mollik' \\ 'Department of Textile Engineering, Bangladesh University of \\ Business and Technology, Bangladesh \\ ${ }^{2}$ Department of Textile Engineering, Jashore University of \\ Science and Technology, Bangladesh \\ ${ }^{3}$ Department of Apparel Engineering, Bangladesh University of \\ Textiles, Bangladesh
}

\begin{abstract}
Correspondence: Tarikul Islam, Department of Textile Engineering, Jashore University of Science and Technology, Jashore-7408, Bangladesh, Tel +8801722202322,

Email matarekul.islam2014@gmail.com
\end{abstract}

Received: May 20, 2019 | Published: June 03, 2019

\section{Introduction}

Achieving higher class performance consent improvement is must and it is a perpetual process. In early age manufacturing word was ruled by economies and businessmen believed that money could be made only by mass production and full utilization of plant capacity which resulted in inflexible plants that was suddenly unchangeable and were accompanied by swollen raw material work in process and finished goods inventories. ${ }^{1}$ For ensuring flexibility, elimination of excess inventory shortened lead time and higher quality of product and customer service, "World Class Manufacturing" and "Lean Manufacturing" terms were introduced by the analysis. ${ }^{2}$ As many manufacturing firms were struggling to implement lean production, industry leaders were looking for designing a new paradigm for shining manufacturing enterprise in $21^{\text {st }}$ century.

In 1991, a new concept was developed through a study participated by 150 industry executives, which study generated a report titled " $21^{\text {st }}$ Century Manufacturing Enterprise Strategy". In consequence of this study the new concept of agile manufacturing was introduced. ${ }^{2-5}$

Lean Manufacturing and Agile Manufacturing are different from each other. Lean manufacturing is a response to probable pressures with limited is a response to complexity occurred by constant change. ${ }^{1}$ Agility is struggle in changeable conditions. ${ }^{4}$ So, agility is an essential part of the industries or enterprises to struggle and be in the competition. In our project work we have focused on the increment of agility in the production so that necessary actions can be implementing rapidly for smart solutions. Information management is one of the vital points in this purpose. Information Systems for the Fashion and Apparel Industry brings together trends and developments in fashion information systems, industrial case-studies, and insights from an international team of authors. The fashion and apparel industry is fast-growing and highly influential. Computerized information systems are essential to support fashion business operations and recent developments in social media, mobile commerce models, radio frequency identification (RFID) technologies, and ERP systems are all driving innovative business measures in the industry.

After an introductory chapter outlining key decision points and information requirements in fast fashion supply chains, Part one focuses on the principles of fashion information systems, with chapters covering how decision making in the apparel supply chains can be improved through the use of fuzzy logic, RFID technologies, evolutionary optimization techniques, and artificial neural networks. Part Two then reviews the range of applications for information systems in the fashion and apparel industry to improve customer choice, aid design, implement intelligent forecasting and procurement systems, and manage inventory and returns.

\section{Materials and methods}

\section{Materials}

In this project, the following materials are used to carry out the data collection and analysis. These are:

1. Stop watch.

2. Pen

3. Paper

4. Pencil

5. Calculator

6. Scale

\section{Methodology}

The main aim of this study is to increase agility of garment industry in sewing department. There is lot of problems like machine breakdown; imperfect operator, absenteeism of operator, imperfect procedure of work etc. can be happened in the sewing floor which hampers the required speed of the work flow in the sewing floor. So, agility in the sewing floor is must. Industrial engineering departments of garment industries are doing their job relentlessly to increase the productivity and improving the quality. But, agility in the production floor is another issue as quick response can accelerate the motion of work. If necessary actions can be actuated within short time then the expected result can change the production level towards the high level. For this study a $100 \%$ export oriented garment industry "Temakaw Fashion Ltd." was selected. The study has been done in the sewing department with the help of IE department. Data has been collected 
from the sewing floor through stop watch. To contrast the agility of IE department, data has been collected two times. First data before implementing "Line wise visual operator status" and "Skill matrix" two excel files and second data after implementing these two files for increasing agility. The total working methods has been pointed into some points in the following:

a. First of all, for the study a $100 \%$ export oriented garment industry was required. "Temakaw Fashion Ltd." A 100\% export oriented garment industry was selected which is situated at Mirpur, Dhaka-1216, Bangladesh.

b. After the selection of factory, the IE department and a sewing line were selected for collecting data. Firstly, total time required from identifying problems in the sewing line to the decision making for solution of the problems was recorded. For collecting these data, within 8 working hour first, second and the hour after lunch were selected. Five days long data has been collected and average value has been taken for analysis. Data has been collected within $11^{\text {th }}$ December 2018 to $11^{\text {th }}$ February 2019.

c. After collecting data, two files named "Line wise visual operator status" and "Skill matrix" was implemented. "Line wise visual operator status" is updated every after each hour by line in charge of each sewing line. Then updated condition of whole sewing floor is accumulated by management trainee officer and updated condition of whole sewing floor is accumulated by IE senior executive and then the collected information is passed to the IE manager for solution of the problems.

\section{Line wise visual operator status}

From Figure 1, it is shown that, it is representing the framework of "Line wise visual operator status" excel file. It could be described as the file is updated every after hour. From the updated file it would be informed about absenteeism, imperfect operator and low efficient operator. After being informed from the particular file to the correspondent person or department would be able to take necessary action according to problems like absenteeism, imperfect operator and low efficient operator. Then through "Line wise visual operator status" file necessary actions (Train up, replacement of operator, work study etc.) will be applied. Due to application of this file the time consumption of the whole process will be reduced.

\section{Line wise visual operator's status}

From Figure 2, it shows a production line of "Temakaw Fashion Ltd". The number of production line is 3 and total number of operator is 46 . In the line there are 41 operators marked as green as they are doing their operation perfectly and efficiently. 2 operators are marked as red as they are imperfect operator for the operations which have been assigned on them. And, 3 operators are marked as yellow as they are doing their work but not perfectly which mean amount of defect is more in their work. The names of the operators are also mentioned in the figure so that right action could be taken for their improvement and also for the improvement of production depending on past records included in the "Skill matrix" excel file.

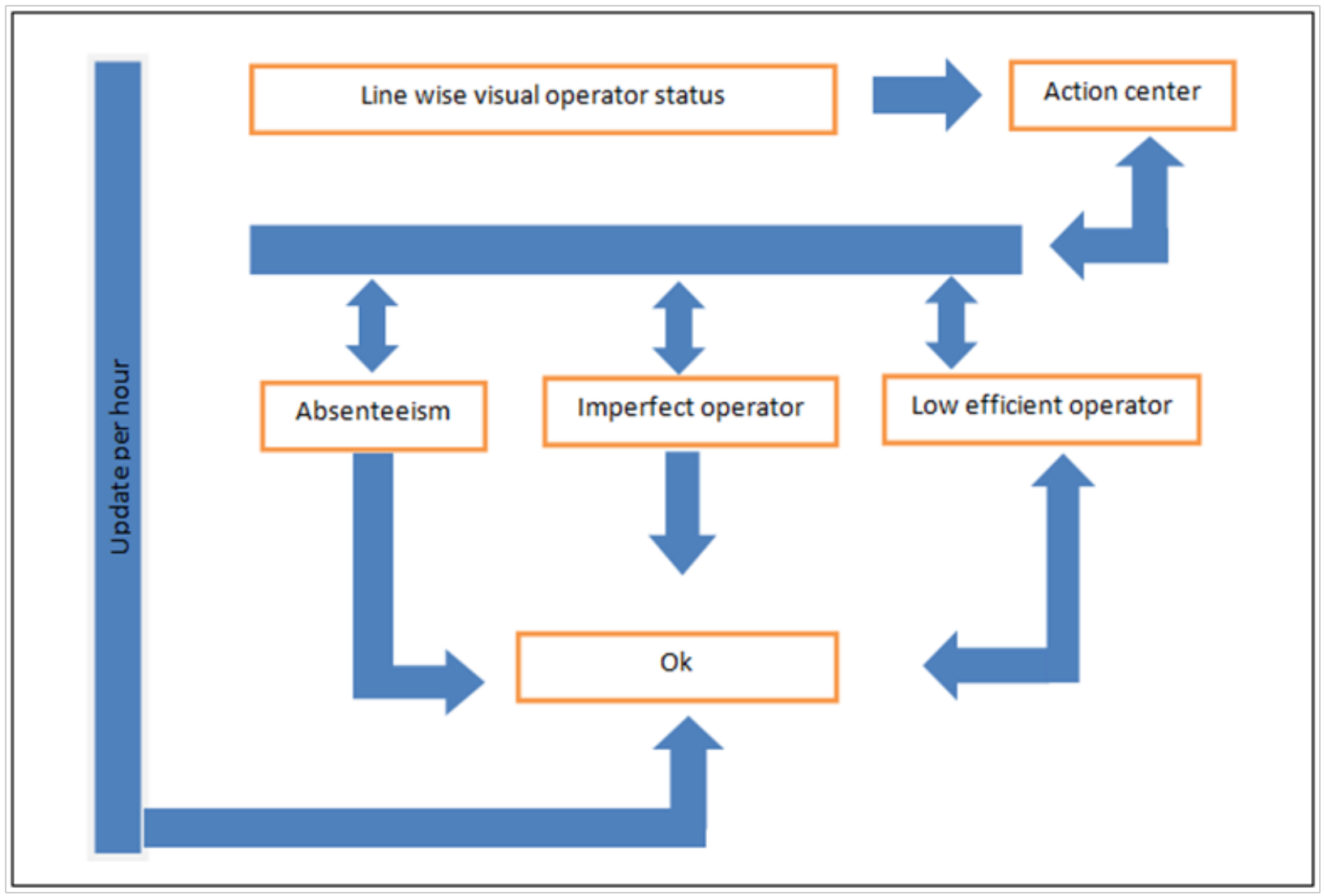

Figure I Line wise visual operator status. 


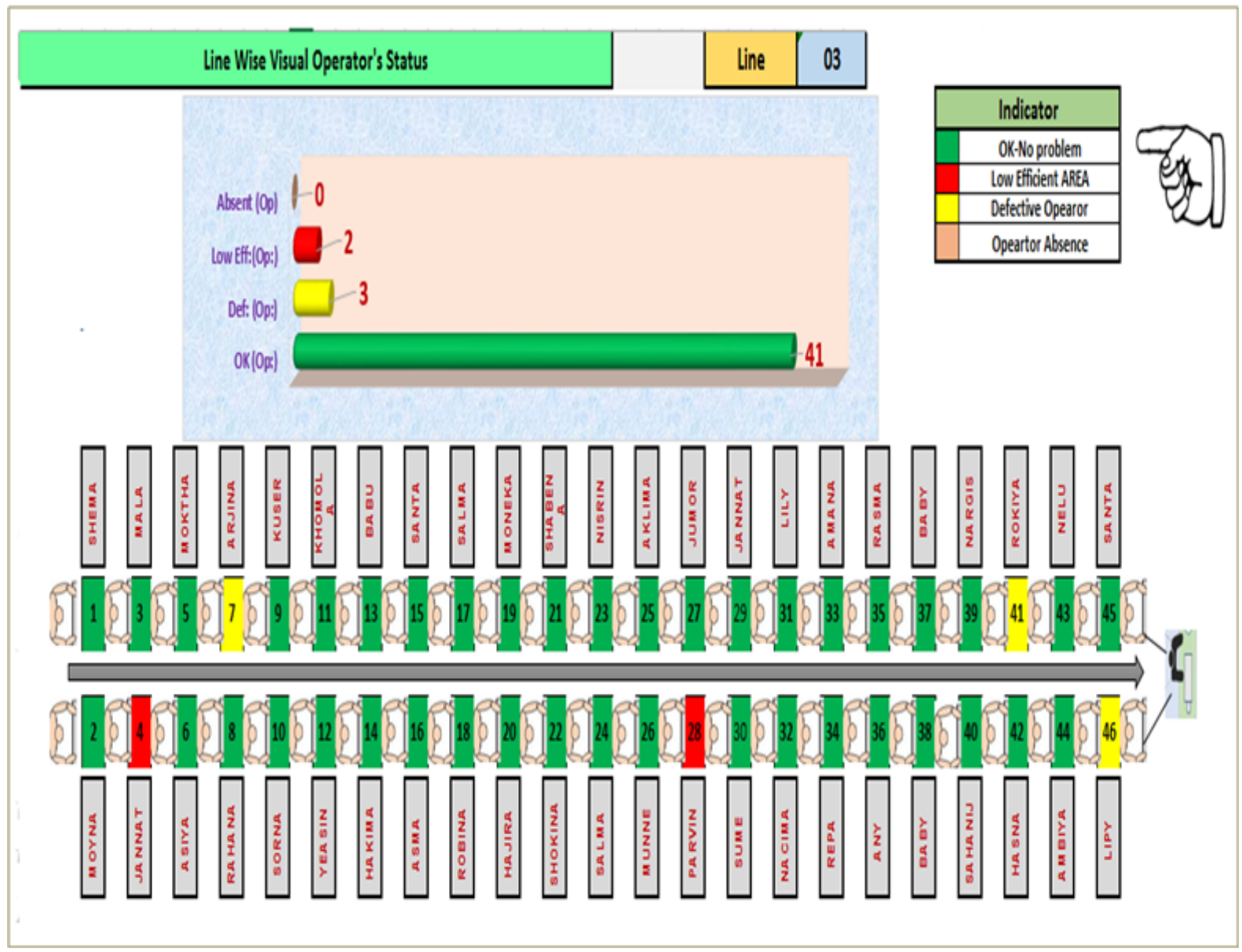

Figure 2 Line wise visual operator's status.

\section{Skill matrix}

Skill matrix is an excel file where a matrix has been developed depending on two years data collected from IE department. In this matrix, operator is categorized on the basis of their efficiency and sewing processes. Through this matrix it can be found out the right operator for required process. Through this file the IE manager can find out the right replacement for absenteeism and low efficient operator. So, "Line wise visual operator status" and "Skill matrix" both files are interrelated.

From Figure 3, it shows the records of the operators of "Temakaw Fashion Ltd". The operator's product quality, efficiency, category of operator, capacity, particular area of working, position in the industry, salary of the operator and date of joining all the information are included here. Like, an operator named Hasina Begum can operate over lock sewing machine and flat lock sewing machine. Her particular area of works are Neck joint, sleeve joint, side seam, bottom hem, shoulder joint, neck top stitch and sleeve hem. Her product quality grade is A and as operator her quality grade is also A. Her capacity is also included in the file according to operation. Her efficiency is $81 \%$ and salary is 6050 taka. The position of her in the industry is Junior Sewing Machine Operator. Like this way, all information of the existing operators are included in "Skill Matrix" excel file. So, anyone can find out right replacement from this file easily.

\section{Data Collection}

i. After implementation of two files secondly data was collected in the same manner described in point number 2 .

ii. On the accomplishment of data collection, the contrast of time before and after implementation of two suggested excel files has been observed through bar diagram to find out the increment of the agility of sewing line.

iii. The time has been recorded in second then it was converted to minutes. Like, in data collection table 1, the first datum of LI (Line in charge)-MTO (management trainee officer) is 15.2 minutes. This datum was found in second 912. This then converted to minutes by dividing 60 . The whole method is shown in the following,

$912 \mathrm{~s}=(912 \div 60)=15.2$ minutes.

In this way, all data has been collected. 
Figure 3 Skill matrix.

\begin{tabular}{|c|c|c|c|c|c|c|c|c|c|c|c|c|c|}
\hline $\begin{array}{l}\text { Line } \\
\text { Info }\end{array}$ & $\begin{array}{l}\text { ID } \\
\text { No. }\end{array}$ & Name & $\begin{array}{l}\text { M/C } \\
\text { Name }\end{array}$ & $\begin{array}{l}\text { Pro. } \\
\text { Grade }\end{array}$ & $\begin{array}{l}\text { Process } \\
\text { Name }\end{array}$ & $\begin{array}{l}\text { Capacity } \\
\text { as per IE }\end{array}$ & Standard & $\begin{array}{l}\text { Effi } \\
(\%)\end{array}$ & $\begin{array}{l}\text { Joining } \\
\text { Date }\end{array}$ & Designation & Grade & $\begin{array}{l}\text { Present } \\
\text { Salary }\end{array}$ & $\begin{array}{l}\text { Proposed } \\
\text { Salary }\end{array}$ \\
\hline 2 & 1 & $\begin{array}{l}\text { Hasina } \\
\text { Begum }\end{array}$ & $\mathrm{OL}$ & A & $\begin{array}{l}\text { Neck } \\
\text { Joint }\end{array}$ & 202 & 250 & $81 \%$ & $\begin{array}{l}12 \text {-Jan- } \\
12\end{array}$ & $\begin{array}{l}\text { Jr. Sewing Machine } \\
\text { Operator }\end{array}$ & A & 6050 & \\
\hline 2 & 1 & $\begin{array}{l}\text { Hasina } \\
\text { Begum }\end{array}$ & $\mathrm{OL}$ & A & $\begin{array}{l}\text { Sleeve } \\
\text { Joint (2) }\end{array}$ & 136 & 167 & $81 \%$ & $\begin{array}{l}12-\mathrm{Jan}- \\
12\end{array}$ & $\begin{array}{l}\text { Jr. Sewing Machine } \\
\text { Operator }\end{array}$ & A & 6050 & \\
\hline 2 & 1 & $\begin{array}{l}\text { Hasina } \\
\text { Begum }\end{array}$ & $\mathrm{OL}$ & A & $\begin{array}{l}\text { Side } \\
\text { Seam }\end{array}$ & 127 & 158 & $80 \%$ & $\begin{array}{l}12-\mathrm{Jan}- \\
12\end{array}$ & $\begin{array}{l}\text { Jr. Sewing Machine } \\
\text { Operator }\end{array}$ & $A$ & 6050 & \\
\hline 2 & 1 & $\begin{array}{l}\text { Hasina } \\
\text { Begum }\end{array}$ & $\mathrm{OL}$ & C & $\begin{array}{l}\text { Shoulder } \\
\text { Joint (2) }\end{array}$ & 160 & 188 & $85 \%$ & $\begin{array}{l}12-\mathrm{Jan}- \\
12\end{array}$ & $\begin{array}{l}\text { Jr. Sewing Machine } \\
\text { Operator }\end{array}$ & $A$ & 6050 & \\
\hline 2 & 1 & $\begin{array}{l}\text { Hasina } \\
\text { Begum }\end{array}$ & $\mathrm{FL}$ & A & $\begin{array}{l}\text { Bottom } \\
\text { Hem }\end{array}$ & 277 & 300 & $92 \%$ & $\begin{array}{l}12-\mathrm{Jan}- \\
12\end{array}$ & $\begin{array}{l}\text { Jr. Sewing Machine } \\
\text { Operator }\end{array}$ & A & 6050 & \\
\hline 2 & 1 & $\begin{array}{l}\text { Hasina } \\
\text { Begum }\end{array}$ & $\mathrm{FL}$ & A & $\begin{array}{l}\text { Front } \\
\text { Neck } \\
\text { T/S }\end{array}$ & 244 & 300 & $81 \%$ & $\begin{array}{l}12 \text {-Jan- } \\
12\end{array}$ & $\begin{array}{l}\text { Jr. Sewing Machine } \\
\text { Operator }\end{array}$ & A & 6050 & \\
\hline 2 & 1 & $\begin{array}{l}\text { Hasina } \\
\text { Begum }\end{array}$ & $\mathrm{FL}$ & A & $\begin{array}{l}\text { Sleeve } \\
\text { Hem } \\
\text { (Close) }\end{array}$ & 108 & 133 & $81 \%$ & $\begin{array}{l}12-\mathrm{Jan}- \\
12\end{array}$ & $\begin{array}{l}\text { Jr. Sewing Machine } \\
\text { Operator }\end{array}$ & A & 6050 & \\
\hline 2 & 1 & $\begin{array}{l}\text { Hasina } \\
\text { Begum }\end{array}$ & & & & & & & $\begin{array}{l}12-\mathrm{Jan}- \\
12\end{array}$ & $\begin{array}{l}\text { Jr. Sewing Machine } \\
\text { Operator }\end{array}$ & $A$ & 6050 & \\
\hline 2 & 1 & $\begin{array}{l}\text { Hasina } \\
\text { Begum }\end{array}$ & & & & & & & $\begin{array}{l}12-\mathrm{Jan}- \\
12\end{array}$ & $\begin{array}{l}\text { Jr. Sewing Machine } \\
\text { Operator }\end{array}$ & A & 6050 & \\
\hline 2 & 1 & $\begin{array}{l}\text { Hasina } \\
\text { Begum }\end{array}$ & & & & & & & $\begin{array}{l}12-\mathrm{Jan}- \\
12\end{array}$ & $\begin{array}{l}\text { Jr. Sewing Machine } \\
\text { Operator }\end{array}$ & A & 6050 & \\
\hline 9 & 2 & $\begin{array}{l}\text { Rozina } \\
\text { Akter }\end{array}$ & Plain & B & $\begin{array}{l}\text { Lower } \\
\text { Placket } \\
\text { Close }\end{array}$ & 118 & 158 & $75 \%$ & $\begin{array}{l}|I-J u|- \\
13\end{array}$ & $\begin{array}{l}\text { Jr. Sewing Machine } \\
\text { Operator }\end{array}$ & B & 5800 & \\
\hline 9 & 2 & $\begin{array}{l}\text { Rozina } \\
\text { Akter }\end{array}$ & Plain & B & $\begin{array}{l}\text { Placket } \\
1 / 16 \mathrm{~T} / \mathrm{S}\end{array}$ & 119 & 158 & $75 \%$ & $\begin{array}{l}11-J u l- \\
13\end{array}$ & $\begin{array}{l}\text { Jr. Sewing Machine } \\
\text { Operator }\end{array}$ & B & 5800 & \\
\hline 9 & 2 & $\begin{array}{l}\text { Rozina } \\
\text { Akter }\end{array}$ & Plain & B & $\begin{array}{l}\text { Placket } \\
\text { Attach }\end{array}$ & 114 & 150 & $76 \%$ & $\begin{array}{l}\mid I-J u l- \\
13\end{array}$ & $\begin{array}{l}\text { Jr. Sewing Machine } \\
\text { Operator }\end{array}$ & B & 5800 & \\
\hline 9 & 2 & $\begin{array}{l}\text { Rozina } \\
\text { Akter }\end{array}$ & OL & B & $\begin{array}{l}\text { Sleeve } \\
\text { Joint (2) }\end{array}$ & 116 & 167 & $69 \%$ & $\begin{array}{l}1 \mid-J u l- \\
13\end{array}$ & $\begin{array}{l}\text { Jr. Sewing Machine } \\
\text { Operator }\end{array}$ & B & 5800 & \\
\hline 9 & 2 & $\begin{array}{l}\text { Rozina } \\
\text { Akter }\end{array}$ & $\mathrm{OL}$ & B & $\begin{array}{l}\text { Side } \\
\text { Seam }\end{array}$ & $12 \mid$ & 158 & $77 \%$ & $\begin{array}{l}1|-J u|- \\
13\end{array}$ & $\begin{array}{l}\text { Jr. Sewing Machine } \\
\text { Operator }\end{array}$ & B & 5800 & \\
\hline 9 & 2 & $\begin{array}{l}\text { Rozina } \\
\text { Akter }\end{array}$ & OL & B & Inseam & 126 & 167 & $75 \%$ & $\begin{array}{l}\mid 1-\text {-Jul- } \\
13\end{array}$ & $\begin{array}{l}\text { Jr. Sewing Machine } \\
\text { Operator }\end{array}$ & B & 5800 & \\
\hline 9 & 2 & $\begin{array}{l}\text { Rozina } \\
\text { Akter }\end{array}$ & $\mathrm{OL}$ & C & $\begin{array}{l}\text { Shoulder } \\
\text { Joint (2) }\end{array}$ & 144 & 188 & $77 \%$ & $\begin{array}{l}1 \mathrm{I}-\mathrm{Jul}- \\
13\end{array}$ & $\begin{array}{l}\text { Jr. Sewing Machine } \\
\text { Operator }\end{array}$ & B & 5800 & \\
\hline $9 \mathrm{k}$ & 2 & $\begin{array}{l}\text { Rozina } \\
\text { Akter }\end{array}$ & & & & & & & $\begin{array}{l}1 \mathrm{I}-\mathrm{Jul}- \\
13\end{array}$ & $\begin{array}{l}\text { Jr. Sewing Machine } \\
\text { Operator }\end{array}$ & B & 5800 & \\
\hline 9 & 2 & $\begin{array}{l}\text { Rozina } \\
\text { Akter }\end{array}$ & & & & & & & $\begin{array}{l}11-J u \mid- \\
13\end{array}$ & $\begin{array}{l}\text { Jr. Sewing Machine } \\
\text { Operator }\end{array}$ & B & 5800 & \\
\hline 9 & 2 & $\begin{array}{l}\text { Rozina } \\
\text { Akter }\end{array}$ & & & & & & & $\begin{array}{l}|1-J u|- \\
13\end{array}$ & $\begin{array}{l}\text { Jr. Sewing Machine } \\
\text { Operator }\end{array}$ & B & 5800 & \\
\hline 7 & 3 & $\begin{array}{l}\text { Reshma } \\
\text { Begum }\end{array}$ & Plain & A & $\begin{array}{l}\text { Placket } \\
1 / 16 \mathrm{~T} / \mathrm{S}\end{array}$ & 127 & 158 & $80 \%$ & $\begin{array}{l}10- \\
\text { Nov-07 }\end{array}$ & $\begin{array}{l}\text { Jr. Sewing Machine } \\
\text { Operator }\end{array}$ & A & 6068 & \\
\hline 7 & 3 & $\begin{array}{l}\text { Reshma } \\
\text { Begum }\end{array}$ & Plain & A & $\begin{array}{l}\text { Placket } \\
\text { Attach }\end{array}$ & 120 & 150 & $80 \%$ & $\begin{array}{l}10- \\
\text { Nov-07 }\end{array}$ & $\begin{array}{l}\text { Jr. Sewing Machine } \\
\text { Operator }\end{array}$ & A & 6068 & \\
\hline 7 & 3 & $\begin{array}{l}\text { Reshma } \\
\text { Begum }\end{array}$ & OL & A & $\begin{array}{l}\text { Neck } \\
\text { Joint }\end{array}$ & 210 & 250 & $84 \%$ & $\begin{array}{l}10- \\
\text { Nov-07 }\end{array}$ & $\begin{array}{l}\text { Jr. Sewing Machine } \\
\text { Operator }\end{array}$ & A & 6068 & \\
\hline
\end{tabular}

Citation: Khan SA, Islam T, Elahi S, et al.An attempt to increase agility of garment industry.J Textile Eng Fashion Technol. 2019;5(3):154-161. DOI: 10.15406/jteft.2019.05.00196 


\begin{tabular}{|c|c|c|c|c|c|c|c|c|c|c|c|c|}
\hline 7 & 3 & $\begin{array}{l}\text { Reshma } \\
\text { Begum }\end{array}$ & OL & A & $\begin{array}{l}\text { Sleeve } \\
\text { Joint (2) }\end{array}$ & 140 & 167 & $84 \%$ & $\begin{array}{l}10- \\
\text { Nov-07 }\end{array}$ & $\begin{array}{l}\text { Jr. Sewing Machine } \\
\text { Operator }\end{array}$ & A & 6068 \\
\hline 7 & 3 & $\begin{array}{l}\text { Reshma } \\
\text { Begum }\end{array}$ & OL & A & $\begin{array}{l}\text { Side } \\
\text { Seam }\end{array}$ & 134 & 158 & $85 \%$ & $\begin{array}{l}\text { l0- } \\
\text { Nov-07 }\end{array}$ & $\begin{array}{l}\text { Jr. Sewing Machine } \\
\text { Operator }\end{array}$ & A & 6068 \\
\hline 7 & 3 & $\begin{array}{l}\text { Reshma } \\
\text { Begum }\end{array}$ & OL & A & Inseam & 138 & 167 & $83 \%$ & $\begin{array}{l}10- \\
\text { Nov-07 }\end{array}$ & $\begin{array}{l}\text { Jr. Sewing Machine } \\
\text { Operator }\end{array}$ & A & 6068 \\
\hline 7 & 3 & $\begin{array}{l}\text { Reshma } \\
\text { Begum }\end{array}$ & OL & C & $\begin{array}{l}\text { Shoulder } \\
\text { Joint (2) }\end{array}$ & 160 & 188 & $85 \%$ & $\begin{array}{l}10- \\
\text { Nov-07 }\end{array}$ & $\begin{array}{l}\text { Jr. Sewing Machine } \\
\text { Operator }\end{array}$ & A & 6068 \\
\hline 7 & 3 & $\begin{array}{l}\text { Reshma } \\
\text { Begum }\end{array}$ & $\mathrm{FL}$ & A & $\begin{array}{l}\text { Arm } \\
\text { Hole T/S } \\
\text { (2) }\end{array}$ & 157 & 188 & $84 \%$ & $\begin{array}{l}\text { I0- } \\
\text { Nov-07 }\end{array}$ & $\begin{array}{l}\text { Jr. Sewing Machine } \\
\text { Operator }\end{array}$ & A & 6068 \\
\hline 7 & 3 & $\begin{array}{l}\text { Reshma } \\
\text { Begum }\end{array}$ & $\mathrm{FL}$ & A & $\begin{array}{l}\text { Front } \\
\text { Neck } \\
\text { T/S }\end{array}$ & 255 & 300 & $85 \%$ & $\begin{array}{l}\text { I0- } \\
\text { Nov-07 }\end{array}$ & $\begin{array}{l}\text { Jr. Sewing Machine } \\
\text { Operator }\end{array}$ & A & 6068 \\
\hline 7 & 3 & $\begin{array}{l}\text { Reshma } \\
\text { Begum }\end{array}$ & $\mathrm{FL}$ & A & $\begin{array}{l}\text { Neck } \\
\text { Piping }\end{array}$ & 212 & 250 & $85 \%$ & $\begin{array}{l}10- \\
\text { Nov-07 }\end{array}$ & $\begin{array}{l}\text { Jr. Sewing Machine } \\
\text { Operator }\end{array}$ & A & 6068 \\
\hline 6 & 4 & $\begin{array}{l}\text { Mukta } \\
\text { Akter }\end{array}$ & Plain & A & $\begin{array}{l}\text { Placket } \\
\text { I/16T/S }\end{array}$ & 127 & 158 & $80 \%$ & $\begin{array}{l}\text { 2-Mar- } \\
10\end{array}$ & $\begin{array}{l}\text { Jr. Sewing Machine } \\
\text { Operator }\end{array}$ & A & 6118 \\
\hline 6 & 4 & $\begin{array}{l}\text { Mukta } \\
\text { Akter }\end{array}$ & Plain & A & $\begin{array}{l}\text { Placket } \\
\text { Attach }\end{array}$ & 120 & 150 & $80 \%$ & $\begin{array}{l}\text { 2-Mar- } \\
10\end{array}$ & $\begin{array}{l}\text { Jr. Sewing Machine } \\
\text { Operator }\end{array}$ & A & 6118 \\
\hline 6 & 4 & $\begin{array}{l}\text { Mukta } \\
\text { Akter }\end{array}$ & OL & A & $\begin{array}{l}\text { Sleeve } \\
\text { Joint (2) }\end{array}$ & 133 & 167 & $80 \%$ & $\begin{array}{l}\text { 2-Mar- } \\
10\end{array}$ & $\begin{array}{l}\text { Jr. Sewing Machine } \\
\text { Operator }\end{array}$ & A & 6118 \\
\hline 6 & 4 & $\begin{array}{l}\text { Mukta } \\
\text { Akter }\end{array}$ & OL & A & $\begin{array}{l}\text { Side } \\
\text { Seam }\end{array}$ & 126 & 158 & $80 \%$ & $\begin{array}{l}\text { 2-Mar- } \\
10\end{array}$ & $\begin{array}{l}\text { Jr. Sewing Machine } \\
\text { Operator }\end{array}$ & A & 6118 \\
\hline 6 & 4 & $\begin{array}{l}\text { Mukta } \\
\text { Akter }\end{array}$ & OL & C & $\begin{array}{l}\text { Shoulder } \\
\text { Joint (2) }\end{array}$ & 160 & 188 & $85 \%$ & $\begin{array}{l}\text { 2-Mar- } \\
10\end{array}$ & $\begin{array}{l}\text { Jr. Sewing Machine } \\
\text { Operator }\end{array}$ & A & 6118 \\
\hline 6 & 4 & $\begin{array}{l}\text { Mukta } \\
\text { Akter }\end{array}$ & $\mathrm{FL}$ & A & $\begin{array}{l}\text { Arm } \\
\text { Hole T/S } \\
(2)\end{array}$ & 152 & 188 & $81 \%$ & $\begin{array}{l}\text { 2-Mar- } \\
10\end{array}$ & $\begin{array}{l}\text { Jr. Sewing Machine } \\
\text { Operator }\end{array}$ & A & 6118 \\
\hline 6 & 4 & $\begin{array}{l}\text { Mukta } \\
\text { Akter }\end{array}$ & $\mathrm{FL}$ & A & $\begin{array}{l}\text { Bottom } \\
\text { Hem }\end{array}$ & 240 & 300 & $80 \%$ & $\begin{array}{l}\text { 2-Mar- } \\
10\end{array}$ & $\begin{array}{l}\text { Jr. Sewing Machine } \\
\text { Operator }\end{array}$ & A & 6118 \\
\hline 6 & 4 & $\begin{array}{l}\text { Mukta } \\
\text { Akter }\end{array}$ & $\mathrm{FL}$ & A & $\begin{array}{l}\text { Front } \\
\text { Neck } \\
\text { T/S }\end{array}$ & 249 & 300 & $83 \%$ & $\begin{array}{l}\text { 2-Mar- } \\
10\end{array}$ & $\begin{array}{l}\text { Jr. Sewing Machine } \\
\text { Operator }\end{array}$ & A & 6118 \\
\hline 6 & 4 & $\begin{array}{l}\text { Mukta } \\
\text { Akter }\end{array}$ & $\mathrm{FL}$ & A & $\begin{array}{l}\text { Neck } \\
\text { Piping }\end{array}$ & 207 & 250 & $83 \%$ & $\begin{array}{l}\text { 2-Mar- } \\
10\end{array}$ & $\begin{array}{l}\text { Jr. Sewing Machine } \\
\text { Operator }\end{array}$ & A & 6118 \\
\hline 6 & 4 & $\begin{array}{l}\text { Mukta } \\
\text { Akter }\end{array}$ & $\mathrm{FL}$ & A & $\begin{array}{l}\text { Sleeve } \\
\text { Hem } \\
\text { (Close) }\end{array}$ & 106 & 133 & $80 \%$ & $\begin{array}{l}\text { 2-Mar- } \\
10\end{array}$ & $\begin{array}{l}\text { Jr. Sewing Machine } \\
\text { Operator }\end{array}$ & A & 6118 \\
\hline I & 5 & Parvin & Plain & B & $\begin{array}{l}\text { Lower } \\
\text { Placket } \\
\text { Close }\end{array}$ & $|2|$ & 158 & $77 \%$ & $\begin{array}{l}\text { I-Apr- } \\
06\end{array}$ & $\begin{array}{l}\text { Jr. Sewing Machine } \\
\text { Operator }\end{array}$ & B & 5950 \\
\hline I & 5 & Parvin & Plain & B & $\begin{array}{l}\text { Placket } \\
\mathrm{I} / 16 \mathrm{~T} / \mathrm{S}\end{array}$ & 123 & 158 & $78 \%$ & $\begin{array}{l}\text { I-Apr- } \\
06\end{array}$ & $\begin{array}{l}\text { Jr. Sewing Machine } \\
\text { Operator }\end{array}$ & B & 5950 \\
\hline I & 5 & Parvin & Plain & B & $\begin{array}{l}\text { Placket } \\
\text { Attach }\end{array}$ & 118 & 150 & $79 \%$ & $\begin{array}{l}\text { I-Apr- } \\
06\end{array}$ & $\begin{array}{l}\text { Jr. Sewing Machine } \\
\text { Operator }\end{array}$ & B & 5950 \\
\hline I & 5 & Parvin & Plain & B & $\begin{array}{l}\text { Placket } \\
\text { Box } \\
\text { Make }\end{array}$ & 97 & 125 & $78 \%$ & $\begin{array}{l}\text { I-Apr- } \\
06\end{array}$ & $\begin{array}{l}\text { Jr. Sewing Machine } \\
\text { Operator }\end{array}$ & B & 5950 \\
\hline I & 5 & Parvin & OL & B & $\begin{array}{l}\text { Sleeve } \\
\text { Joint (2) }\end{array}$ & 128 & 167 & $77 \%$ & $\begin{array}{l}\text { I-Apr- } \\
06\end{array}$ & $\begin{array}{l}\text { Jr. Sewing Machine } \\
\text { Operator }\end{array}$ & B & 5950 \\
\hline I & 5 & Parvin & OL & B & $\begin{array}{l}\text { Side } \\
\text { Seam }\end{array}$ & 124 & 158 & $78 \%$ & $\begin{array}{l}\text { I-Apr- } \\
06\end{array}$ & $\begin{array}{l}\text { Jr. Sewing Machine } \\
\text { Operator }\end{array}$ & B & 5950 \\
\hline I & 5 & Parvin & $\mathrm{FL}$ & B & $\begin{array}{l}\text { Front } \\
\text { Neck } \\
\mathrm{T} / \mathrm{S}\end{array}$ & 231 & 300 & $77 \%$ & $\begin{array}{l}\text { I-Apr- } \\
06\end{array}$ & $\begin{array}{l}\text { Jr. Sewing Machine } \\
\text { Operator }\end{array}$ & B & 5950 \\
\hline 1 & 5 & Parvin & $\mathrm{FL}$ & B & $\begin{array}{l}\text { Shoulder } \\
\text { T/S }\end{array}$ & 235 & 300 & $78 \%$ & $\begin{array}{l}\text { I-Apr- } \\
06\end{array}$ & $\begin{array}{l}\text { Jr. Sewing Machine } \\
\text { Operator }\end{array}$ & B & 5950 \\
\hline I & 5 & Parvin & $\mathrm{FL}$ & C & $\begin{array}{l}\text { Sleeve } \\
\text { Hem } \\
\text { (Close) }\end{array}$ & 98 & 133 & $74 \%$ & $\begin{array}{l}\text { I-Apr- } \\
06\end{array}$ & $\begin{array}{l}\text { Jr. Sewing Machine } \\
\text { Operator }\end{array}$ & B & 5950 \\
\hline I & 5 & Parvin & & & & & & & $\begin{array}{l}\text { I-Apr- } \\
06\end{array}$ & $\begin{array}{l}\text { Jr. Sewing Machine } \\
\text { Operator }\end{array}$ & B & 5950 \\
\hline
\end{tabular}

Citation: Khan SA, Islam T, Elahi S, et al.An attempt to increase agility of garment industry.J Textile Eng Fashion Technol. 20I9;5(3):I54-I6I . 


\section{Result and discussion}

Data collection table before implementing "Line wise visual operator status" and "Skill matrix" files (Table I) (Table 2).

Table I Data chart

\begin{tabular}{|c|c|c|c|c|c|}
\hline \multirow{2}{*}{$\begin{array}{l}\text { No. of } \\
\text { day }\end{array}$} & \multirow{2}{*}{ Hour } & \multicolumn{3}{|c|}{$\begin{array}{l}\text { Time required for passing } \\
\text { information in ascendance } \\
\text { order ( } \mathrm{min})\end{array}$} & \multirow{2}{*}{$\begin{array}{l}\text { Time for } \\
\text { taking decision } \\
\text { and preparing } \\
\text { action plans } \\
\text { (min) }\end{array}$} \\
\hline & & LI-MTO & $\begin{array}{l}\text { MTO- } \\
\text { Sr. Ex }\end{array}$ & $\begin{array}{l}\text { Sr. Ex- } \\
\text { IE Mng. }\end{array}$ & \\
\hline \multirow{3}{*}{ Day I } & $I^{\text {st }}$ & 15.2 & 16.7 & 13.8 & 15.8 \\
\hline & $2^{\text {nd }}$ & 12.3 & 12.4 & 12.3 & 18.2 \\
\hline & $\begin{array}{l}\text { After } \\
\text { lunch }\end{array}$ & 16.1 & 17.6 & 17.5 & 11.6 \\
\hline \multirow{3}{*}{ Day 2} & $\left.\right|^{\text {st }}$ & 17.2 & 15.2 & 16.3 & 17.4 \\
\hline & $2^{\text {nd }}$ & 11.8 & 13.3 & 12.1 & 10.7 \\
\hline & $\begin{array}{l}\text { After } \\
\text { lunch }\end{array}$ & 14.3 & 16.4 & 15.4 & 11.3 \\
\hline \multirow{3}{*}{ Day 3} & $\left.\right|^{\text {st }}$ & 16.2 & 17.3 & 17.3 & 20.2 \\
\hline & $2^{\text {nd }}$ & 13.4 & 12.2 & 13.6 & 16.4 \\
\hline & $\begin{array}{l}\text { After } \\
\text { lunch }\end{array}$ & 14.7 & 15.2 & 17.1 & 16.6 \\
\hline \multirow{3}{*}{ Day 4} & $I^{\text {st }}$ & 19.3 & 15.9 & 16.2 & 14.8 \\
\hline & $2^{\text {nd }}$ & 13.2 & 14.2 & 14.4 & 13.2 \\
\hline & $\begin{array}{l}\text { After } \\
\text { lunch }\end{array}$ & 15.7 & 16.2 & 14.3 & 19.5 \\
\hline \multirow{3}{*}{ Day 5} & $I^{\text {st }}$ & 17.6 & 16.6 & 16.2 & 14.2 \\
\hline & $2^{\text {nd }}$ & 14.1 & 15.3 & 14.3 & 13.4 \\
\hline & $\begin{array}{l}\text { After } \\
\text { lunch }\end{array}$ & 19.1 & 17.8 & 16.2 & 16.8 \\
\hline \multicolumn{2}{|c|}{ Average time } & $\begin{array}{l}15.35 \\
\min \end{array}$ & $\begin{array}{l}\text { I5.49 } \\
\text { min }\end{array}$ & $\begin{array}{l}15.13 \\
\min \end{array}$ & $15.34 \mathrm{~min}$ \\
\hline
\end{tabular}

LI, line in-charge; MTO, management trainee officer; Sr. Ex, senior executive; IE Mng, industrial engineering manager

Table 2 Data chart 2

\begin{tabular}{|c|c|c|c|c|}
\hline \multirow{2}{*}{$\begin{array}{l}\text { No. of } \\
\text { day }\end{array}$} & \multirow{2}{*}{ Hour } & \multicolumn{3}{|c|}{$\begin{array}{l}\text { Time required for passing information } \\
\text { in descendance order ( } \mathrm{min})\end{array}$} \\
\hline & & $\begin{array}{l}\text { IE } \\
\text { Mng.- } \\
\text { Sr Ex }\end{array}$ & $\begin{array}{l}\text { Sr. Ex- } \\
\text { MTO }\end{array}$ & MTO-LI \\
\hline \multirow{3}{*}{ Day I } & $\left.\right|^{\text {st }}$ & 4.6 & 4.5 & 5.6 \\
\hline & $2^{\text {nd }}$ & 5.1 & 4.8 & 4.6 \\
\hline & $\begin{array}{l}\text { After } \\
\text { lunch }\end{array}$ & 5 & 4.8 & 4.8 \\
\hline \multirow{3}{*}{ Day 2} & $\left.\right|^{\text {st }}$ & 4.9 & 5.2 & 4.9 \\
\hline & $2^{\text {nd }}$ & 5.4 & 4.6 & 4.5 \\
\hline & $\begin{array}{l}\text { After } \\
\text { lunch }\end{array}$ & 4.6 & 4.6 & 4.6 \\
\hline \multirow{3}{*}{ Day 3} & $I^{\text {st }}$ & 4.8 & 5.2 & 5 \\
\hline & $2^{\text {nd }}$ & 5.1 & 4.9 & 4.8 \\
\hline & $\begin{array}{l}\text { After } \\
\text { lunch }\end{array}$ & 4.9 & 5 & 4.8 \\
\hline
\end{tabular}

\begin{tabular}{|c|c|c|c|c|}
\hline \multirow{2}{*}{$\begin{array}{l}\text { No. of } \\
\text { day }\end{array}$} & \multirow[b]{2}{*}{ Hour } & \multicolumn{3}{|c|}{$\begin{array}{l}\text { Time required for passing information } \\
\text { in descendance order ( } \mathrm{min})\end{array}$} \\
\hline & & $\begin{array}{l}\text { IE } \\
\text { Mng.- } \\
\text { Sr Ex }\end{array}$ & $\begin{array}{l}\text { Sr. Ex- } \\
\text { MTO }\end{array}$ & MTO-LI \\
\hline \multirow{3}{*}{ Day 4} & $\left.\right|^{\text {st }}$ & 4.6 & 4.4 & 4.3 \\
\hline & $2^{\text {nd }}$ & 5 & 4.7 & 4.6 \\
\hline & $\begin{array}{l}\text { After } \\
\text { lunch }\end{array}$ & 4.6 & 5.4 & 5.2 \\
\hline \multirow{3}{*}{ Day 5} & $I^{\text {st }}$ & 5.3 & 4.8 & 4.6 \\
\hline & $2^{\text {nd }}$ & 4.8 & 4.9 & 5 \\
\hline & $\begin{array}{l}\text { After } \\
\text { lunch }\end{array}$ & 4.8 & 5.3 & 5.1 \\
\hline \multicolumn{2}{|c|}{ Average time } & $4.6 \mathrm{mi}$ & $4.87 \mathrm{mi}$ & $4.83 \mathrm{~min}$ \\
\hline
\end{tabular}

LI, line in-charge; MTO, management trainee officer; Sr. Ex, senior executive; IE Mng, industrial engineering manager

Data collection table after implementing "Line wise visual operator status" and "Skill matrix" files (Table 3) (Table 4).

Table 3 Data chart 3

\begin{tabular}{|c|c|c|c|c|c|}
\hline \multirow{2}{*}{$\begin{array}{l}\text { No. of } \\
\text { day }\end{array}$} & \multirow{2}{*}{ Hour } & \multicolumn{3}{|c|}{$\begin{array}{l}\text { Time required for } \\
\text { passing information in } \\
\text { ascendance order ( } \mathrm{min})\end{array}$} & \multirow{2}{*}{$\begin{array}{l}\text { Time for } \\
\text { preparing } \\
\text { action plans } \\
\text { (min) }\end{array}$} \\
\hline & & $\begin{array}{l}\text { LI- } \\
\text { MTO }\end{array}$ & $\begin{array}{l}\text { MTO- } \\
\text { Sr. Ex }\end{array}$ & $\begin{array}{l}\text { Sr. Ex- } \\
\text { IE Mng. }\end{array}$ & \\
\hline \multirow{3}{*}{ Day I } & $\left.\right|^{\text {st }}$ & 4.9 & 4.9 & 4.8 & 19.1 \\
\hline & $2^{\text {nd }}$ & 5.1 & 5.2 & 4.7 & 20.5 \\
\hline & $\begin{array}{l}\text { After } \\
\text { lunch }\end{array}$ & 4.6 & 4.7 & 4.7 & 20.4 \\
\hline \multirow{3}{*}{ Day 2} & $I^{s t}$ & 4.9 & 4.9 & 4.8 & 15.2 \\
\hline & $2^{\text {nd }}$ & 5.3 & 4.6 & 4.9 & 14.1 \\
\hline & $\begin{array}{l}\text { After } \\
\text { lunch }\end{array}$ & 5.1 & 5.2 & 5 & 13.5 \\
\hline \multirow{3}{*}{ Day 3} & $\left.\right|^{\text {st }}$ & 4.5 & 4.6 & 4.6 & 13.7 \\
\hline & $2^{\text {nd }}$ & 5.6 & 4.8 & 4.8 & 13.6 \\
\hline & $\begin{array}{l}\text { After } \\
\text { lunch }\end{array}$ & 5.2 & 4.9 & 4.7 & 20.1 \\
\hline \multirow{3}{*}{ Day 4} & $\left.\right|^{\text {st }}$ & 4.8 & 4.9 & 4.6 & 16.2 \\
\hline & $2^{\text {nd }}$ & 4.7 & 4.8 & 4.7 & 12.3 \\
\hline & $\begin{array}{l}\text { After } \\
\text { lunch }\end{array}$ & 5 & 5.2 & 5.1 & 12.8 \\
\hline \multirow{3}{*}{ Day 5} & $\left.\right|^{s t}$ & 4.6 & 4.8 & 4.8 & 11.2 \\
\hline & $2^{\text {nd }}$ & 5.4 & 5.2 & 5.2 & 16.4 \\
\hline & $\begin{array}{l}\text { After } \\
\text { lunch }\end{array}$ & 4.7 & 4.7 & 4.8 & 13.8 \\
\hline \multicolumn{2}{|c|}{ Average time } & $\begin{array}{l}4.65 \\
\mathrm{~min}\end{array}$ & $\begin{array}{l}4.89 \\
\mathrm{~min}\end{array}$ & $\begin{array}{l}4.81 \\
\mathrm{~min}\end{array}$ & $14.1 \mathrm{~min}$ \\
\hline
\end{tabular}

LI, line in-charge; MTO, management trainee officer; Sr. Ex, senior executive; IE Mng, industrial engineering manager 
Table 4 Data chart 4

\begin{tabular}{|c|c|c|c|c|}
\hline \multirow{2}{*}{$\begin{array}{l}\text { No. of } \\
\text { day }\end{array}$} & \multirow{2}{*}{ Hour } & \multicolumn{3}{|c|}{$\begin{array}{l}\text { Time required for passing } \\
\text { information in descendance } \\
\text { order (min) }\end{array}$} \\
\hline & & $\begin{array}{l}\text { IE } \\
\text { Mng.- } \\
\text { Sr Ex }\end{array}$ & $\begin{array}{l}\text { Sr. Ex- } \\
\text { MTO }\end{array}$ & MTO-LI \\
\hline \multirow{3}{*}{ Day I } & $I^{\text {st }}$ & 3.6 & 4.2 & 4.1 \\
\hline & $2^{\text {nd }}$ & 3.9 & 4.3 & 3.9 \\
\hline & $\begin{array}{l}\text { After } \\
\text { lunch }\end{array}$ & 4.2 & 4.5 & 4.5 \\
\hline \multirow{3}{*}{ Day 2} & $I^{\text {st }}$ & 3.6 & 3.8 & 3.7 \\
\hline & $2^{\text {nd }}$ & 3.2 & 3.3 & 3.1 \\
\hline & $\begin{array}{l}\text { After } \\
\text { lunch }\end{array}$ & 3 & 2.9 & 2.7 \\
\hline \multirow{3}{*}{ Day 3} & $\left.\right|^{\text {st }}$ & 3.1 & 2.8 & 2.9 \\
\hline & $2^{\text {nd }}$ & 3.2 & 3.2 & 3 \\
\hline & $\begin{array}{l}\text { After } \\
\text { lunch }\end{array}$ & 4.3 & 4.5 & 4.2 \\
\hline \multirow{3}{*}{ Day 4} & $I^{\text {st }}$ & 3.7 & 3.5 & 3.6 \\
\hline & $2^{\text {nd }}$ & 2.9 & 3.2 & 3.4 \\
\hline & $\begin{array}{l}\text { After } \\
\text { lunch }\end{array}$ & 3.1 & 3.3 & 3.1 \\
\hline \multirow{3}{*}{ Day 5} & $I^{\text {st }}$ & 2.5 & 2.8 & 2.8 \\
\hline & $2^{\text {nd }}$ & 3.5 & 2.4 & 2.6 \\
\hline & $\begin{array}{l}\text { After } \\
\text { lunch }\end{array}$ & 3.2 & 3.6 & 3.5 \\
\hline \multicolumn{2}{|c|}{ Average time } & $3.4 \mathrm{~min}$ & $3.48 \mathrm{~min}$ & $3.4 \mathrm{~min}$ \\
\hline
\end{tabular}

LI, line in-charge; MTO, management trainee officer; Sr. Ex, senior executive; IE Mng, industrial engineering manager

\section{Before implementing the current system}

In the scenario (Figure 4), it shows that line in charge after starting of work of the first hour take the update of the lines and report to the management trainee officer. For this activity it takes 15.35 minutes. After being informed the MTO pass the information of the whole floor to the senior IE executive with written document and the IE executive pass the information of the total floors to the IE manager. For this, it takes 15.49 minutes and 15.13 minutes respectively for both activities. And, IE manager takes 15.34 minutes to do action plan for the relevant problems. It may be replacement of the worker, method study or work study etc. After the completion of the plan IE manager pass the information in the reverse sequence for implementation. And it takes 14.3 minutes in total to start the execution of the plan.

\section{Total time required initiating an action to solve problems in the sewing floor}

$$
\begin{aligned}
& =(15.35+15.49+15.13+15.34+4.6+4.87+4.83) \\
& =75.61 \text { minutes. }
\end{aligned}
$$

From Figure 5, it shows that after the implementation of line wise visual operator status, the total time to start execution of the plan has been minimized. At the early hour the condition of sewing line is updated by the line in charge, then the updated scenario of whole sewing floor is accumulated by management trainee officer by shared computer network in the industry. After that the information of whole sewing department is accumulated by senior executive through shared network. Then the total scenario of sewing department is passed to the IE manger. Then IE the total scenario is observed by the manager to solve the raised problems (absenteeism, low efficient worker, imperfect worker). The required replacements of the workers are done by manager by the help of excel file "Skill matrix" where the data of all recruited workers are being preserved.

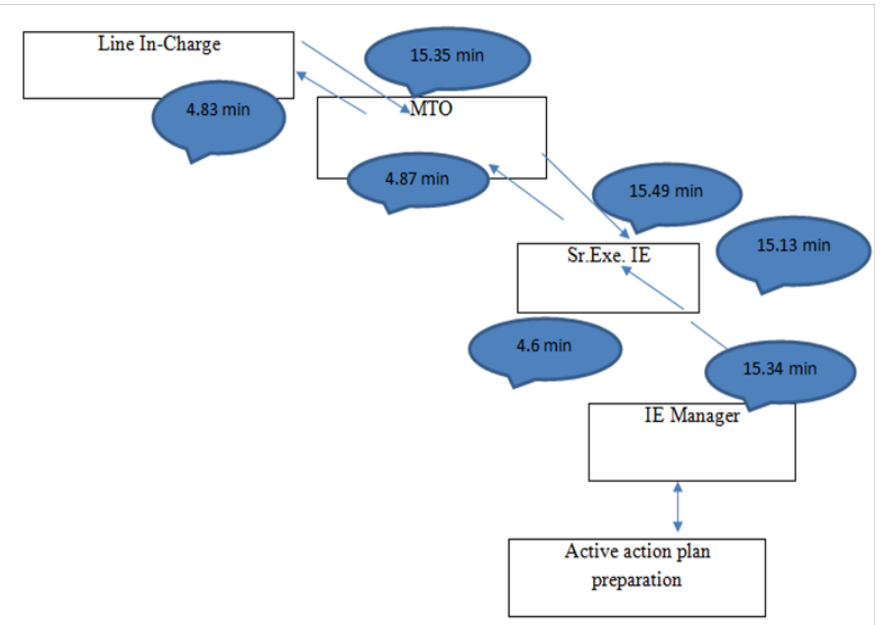

Figure 4 Before implementing the suggested excel files.

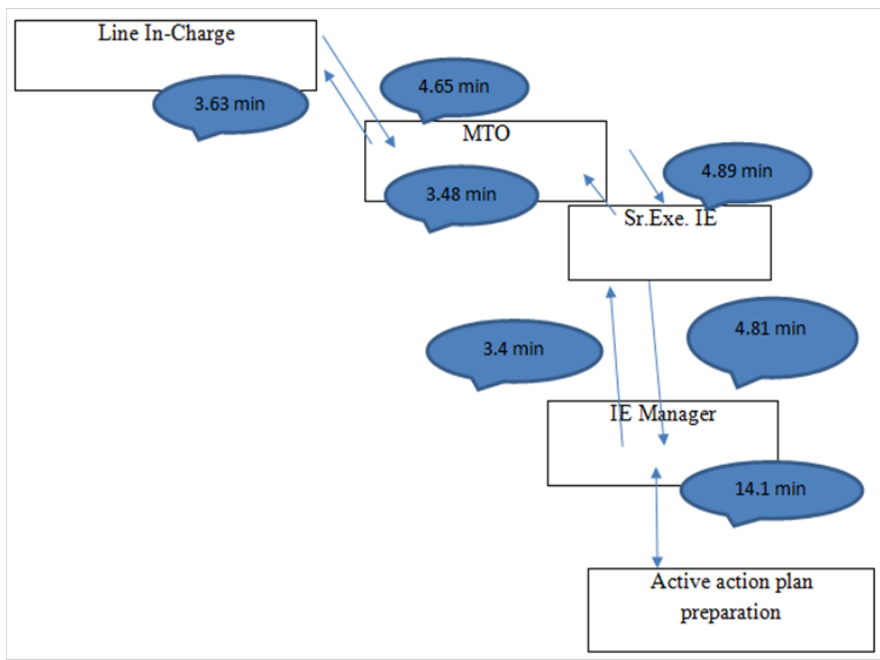

Figure 5 After Applying suggested excel files.

Total time required initiating an action to solve problems in the sewing floor

$$
\begin{aligned}
& =(4.65+4.89+4.81+14.1+3.4+3.48+3.4) \\
& =38.73 \text { minutes. }
\end{aligned}
$$

\section{So, total time savings for taking action against problem}

$=(75.61 \mathrm{~min}-38.73 \mathrm{~min})$

$=36.87$ minutes.

So, we are being able to take initiation 36.87 minutes faster than the previous systems which represent the agility of the industry.

From Figure 6, it shows that before the system it's takes 75.61 
minutes but Right now it takes only 38.73 minutes. The system has been saving 36.87 minutes of our time. ${ }^{5-11}$

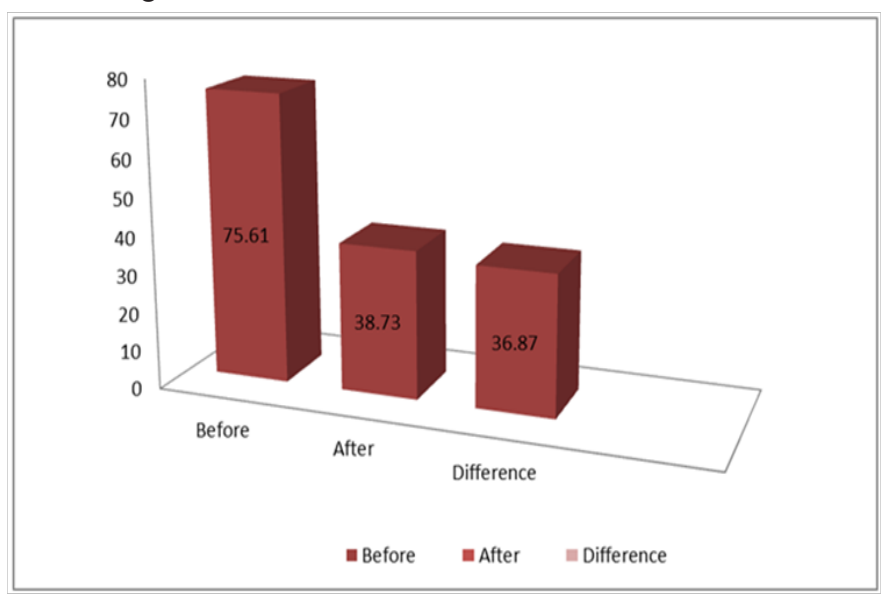

Figure 6 Graphical status of the system.

\section{Conclusion}

This Project work is done to know the influence of MIS in garments industry and its impact in every section of the industry. Moreover, to collect all necessary information a lot of efforts were taken but it is true that within this short time it is quite impossible to achieve $100 \%$ success. Besides, in this research work there were some limitations. Here, only three types of problem have been taken into consideration. They were absenteeism, imperfection in operation and low efficiency in work. Without these three problems there are also several problems like machine break down, bottleneck etc. These problems should be taken in the research. But, work has been continued to improve the two excel files for including the remaining problems in the sewing lines. Such kind of work is new in the garment industry and the method only has been applied in sewing section. So, process is under investigation to apply the method both in cutting and finishing sections also. If further work will be done on this track it would be appreciated.

\section{Author's contribution}

The work has been done under the supervision of S.A.K. The work design, data collection, data analysis and presentation are done by M.N.S and M.M.M. with the help of T.I. and finally all authors revised the manuscript.

\section{Acknowledgments}

Authors acknowledge the authority and management of Temakaw Fashion Ltd. Mirpur, Dhaka-1216, Bangladesh especially the Industrial Engineering department for performing the project work and continuous support.

\section{Conflicts of interest}

The authors declare that they have no competing interests.

\section{References}

1. Sanchez LM, Nagi R. A review of agile manufacturing systems. International Journal of Production Research. 2001;39(16):3561-3600.

2. Sheridan JH. Agile manufacturing: Stepping beyond lean production. Industry Week. 1993;242(8):30-46.

3. Strader TJ, Lin FR, Shaw MJ. Information infrastructure for electronic virtual organization management. Decision Support Systems. 1998;23(1):75-94.

4. Richards CW. Agile manufacturing: beyond lean? Production and Inventory Management Journal. 1996;37(2):60-64.

5. Montreuil B, Venkatadri U, Rardin RL. Fractal layout organization for job shop environments. International Journal of Production Research. 1999;37(3):501-521.

6. Canel C, Khumawala BM. Multi-period international facilities location: an algorithm and application. International Journal of Production Research. 1997;35(7):1891-1910.

7. Haug P. International location and production transfer model for high technology multinational enterprises. International Journal of Production Research. 1992;30(3):559-572.

8. Meller RD, Mungwattana A. Multi-shuttle automated storage/retrieval systems. IIE Transactions. 1997;29(10):925-938.

9. Mills JJ. An integrated infrastructure for agile manufacturing. Manufacturing Science and Engineering, ASME International Mechanical Engineering Congress and Exposition, San Francisco; 1995:1037-1047.

10. Govindaraj T. A distributed information system to assist engineering designers. IERC Proceedings 1997, 6th Annual Industrial Engineering Research Conference. GA, USA: Norcross; 1997:537-542.

11. Aoyama M. A web-based agile software development. IEEE Software. $1998 ; 15(6): 56-65$. 